\title{
A multidimensional hydrodynamic code for structure evolution in cosmology
}

\author{
Vicent Quilis, José M므. Ibáñez \\ and \\ Diego Sáez \\ Departament d'Astronomia i Astrofísica, Universitat de València, E-46100 Burjassot, \\ València, Spain
}

Received __; accepted _ 


\begin{abstract}
A cosmological multidimensional hydrodynamic code is described and tested. This code is based on modern high-resolution shock-capturing techniques. It can make use of a linear or a parabolic cell reconstruction as well as an approximate Riemann solver. The code has been specifically designed for cosmological applications. Two tests including shocks have been considered: the first one is a standard shock tube and the second test involves a spherically symmetric shock. Various additional cosmological tests are also presented. In this way, the performance of the code is proved. The usefulness of the code is discussed; in particular, this powerful tool is expected to be useful in order to study the evolution of the hot gas component located inside nonsymmetric cosmological structures.
\end{abstract}

Subject headings: hydrodynamics - methods: numerical - large-scale structure of Universe - Cosmology 


\section{Introduction}

In this paper, a new hydrodynamical code is presented and tested. This code is the multidimensional extension of a previous one described by Quilis et al.(1994); it has been designed with the essential aim of simulating the evolution of a nonsymmetric three-dimensional (3D) distribution of gas. The features of this distribution are similar to those of the hot rarified gas located inside cosmological structures. Further applications of the multidimensional code will be presented elsewhere.

Even if the numerical techniques, the physical principles and the equations involved in a code are appropriate, the hydrodynamical code must be tested in order to discover unexpected failures and possible limitations. Six tests have been selected spanning a significant set of situations which allow us to check all the relevant ingredients of the code. The chosen tests correspond to six problems with known analytical or numerical solutions. These solutions are compared with those obtained using our multidimensional code. Results are encouraging.

Two types of methods can be used in order to study structure formation in Cosmology: Analytical and numerical methods. Among the analytical methods, several Eulerian and Lagragian hydrodynamical approaches have been proposed. In these approaches, the Universe is considered as a fluid (see Bertschinger 1991). Let us mention, as examples, the Zel'dovich solution (Zel'dovich 1970), the Adhesion model (Gurbatov et al. 1989), and the frozen flow approximation (Matarrese et al. 1992). These approaches apply beyond the linear regime, but they have limitations. Zel'dovich's 3D solution only works properly up to the mildly nonlinear regime and both the frozen flow and the adhesion models introduce fictitious forces in order to avoid caustic formation. On account of these limitations, it

seems that the use of other techniques such as the numerical ones is appropriate. N-body simulations are used in the pressureless case and very robust hydrodynamical codes have 
been proposed for studying collisional matter. Let us mention the code developed by Cen (1992), which uses artificial viscosity, and the smooth particle hydrodynamic codes originally developed by Gingold \& Monaghan (1977) and Lucy (1977), independently. This paper is a preliminary study, in which modern numerical techniques are implemented in order to design a code for future applications to structure formation in Cosmology.

We have built up a multidimensional cosmological hydrodynamical code based on modern high-resolution shock-capturing (HRSC) techniques. These HRSC methods were specifically designed for solving hyperbolic systems of conservation laws and have two main features: they are at least second order accurate on the smooth part of the flow and they give well resolved nonoscillatory discontinuities (LeVeque 1992). By construction HRSC schemes avoid using numerical artifacts, such as the artificial viscosity, in order to smear shocks. With HRSC techniques strong shocks are sharply solved, typically, in two or three numerical cells, and they are free of spurious oscillations due to the Gibbs phenomenon. This last property could be of crucial importance in 3D calculations where the numerical grid is constrained for obvious technical reasons and has a poor resolution.

In recent years, HRSC methods have been applied widely in many astrophysical fields: interacting stellar winds (see, e.g., Mellema et al. 1991), type II supernovae explosions (see, e.g., Müller 1994), relativistic jets (Martí et al., 1995), etc. More recently, some codes -with cosmological applications- based on HRSC techniques have been presented. A very recent multidimensional cosmological hydro-code built up using these techniques is the one described by Ryu et al. (1993). In Quilis et al. (1994) we analyzed the main features of a one-dimensional code, and in the present paper we are concerned with the multidimensional version of this code.

Hereafter, $t$ stands for the cosmological time, $t_{0}$ is the age of the Universe, $a(t)$ is the scale factor of a flat background. $\dot{X}$ stands for the derivative of the function $X$ with respect 
to the cosmological time. Function $\dot{a} / a$ is denoted by $H$. Hubble constant is the present value of $H$; its value in units of $100 \mathrm{Km} \mathrm{s}^{-1} \mathrm{Mpc}^{-1}$ is $h$. Velocities are given in units of the speed of light. $\rho$ and $\rho_{B}$ stand for mass density and background mass density, respectively. The density contrast is $\delta=\left(\rho-\rho_{B}\right) / \rho_{B}$. The background is flat. $p$ and $\epsilon$ stand for pressure and internal energy per unit mass, respectively.

The plan of this paper is as follows: In Section 2, our numerical code is described. In Section 3, the results of several tests are shown. Finally, a general discussion is presented in Section 4 .

\section{Equations and numerical procedure}

In this Section we are going to write the basic equations governing the evolution of cosmological inhomogeneities as a hyperbolic system of conservation laws. The mathematical properties of this kind of system have been well studied (see, e.g., Lax 1973). Numerical algorithms have been specifically designed for solving these systems of partial differential equations (see, e.g., Yee 1989 and LeVeque 1992).

\subsection{Evolution equations in conservation form}

For spatial scales which are small enough (see below), cosmological inhomogeneities evolve according to the following equations (Peebles 1980):

$$
\begin{gathered}
\frac{\partial \delta}{\partial t}+\frac{1}{a} \nabla \cdot(1+\delta) \vec{v}=0 \\
\frac{\partial \vec{v}}{\partial t}+\frac{1}{a}(\vec{v} \cdot \nabla) \vec{v}+H \vec{v}=-\frac{1}{\rho a} \nabla p-\frac{1}{a} \nabla \phi \\
\frac{\partial E}{\partial t}+\frac{1}{a} \nabla \cdot[(E+p) \vec{v}]=-3 H(E+p)-H \rho \vec{v}^{2}-\frac{\rho \vec{v}}{a} \nabla \phi
\end{gathered}
$$




$$
\nabla^{2} \phi=\frac{3}{2} H^{2} a^{2} \delta
$$

where $\vec{x}, \vec{v}=a(t) \frac{d \vec{x}}{d t}=\left(v_{x}, v_{y}, v_{z}\right), E$ and $\phi(t, \vec{x})$ are, respectively, the Eulerian coordinates, the peculiar velocity, the total energy $E=\rho \epsilon+\frac{1}{2} \rho v^{2}\left(v^{2}=v_{x}^{2}+v_{y}^{2}+v_{z}^{2}\right)$, and the peculiar Newtonian gravitational potential. Pressure gradients and gravitational forces are the responsible for this evolution.

This approach applies if the following conditions are satisfied: a) The size of the inhomogeneity is much smaller than the causal horizon size; thus, background curvature is negligible, b) velocities are much smaller than the speed of light, and c) no strong local gravitational fields are present. These conditions make a relativistic approach unnecessary .

In 3D case, Eqs (1), (2) and (3) can be written as follows:

$$
\begin{gathered}
\frac{\partial \delta}{\partial t}+\frac{\partial}{\partial x^{\alpha}}\left[(\delta+1) \frac{v_{\alpha}}{a}\right]=0 \\
\frac{\partial}{\partial t}\left[(\delta+1) v_{\alpha}\right]+\frac{\partial}{\partial x^{\beta}}\left[\frac{v_{\alpha} v_{\beta}(\delta+1)}{a}+\frac{p}{a \rho_{B}} \delta_{\alpha \beta}\right]=-\frac{(\delta+1)}{a} \frac{\partial \phi}{\partial x^{\alpha}}-(\delta+1) v_{\alpha} H \\
\frac{\partial E}{\partial t}+\frac{\partial}{\partial x^{\alpha}}\left[\frac{(E+p) v_{\alpha}}{a}\right]=-3 H(E+p)-(\delta+1) \rho_{B} H v^{2}-\frac{(\delta+1) v_{\alpha} \rho_{B}}{a} \frac{\partial \phi}{\partial x^{\alpha}}
\end{gathered}
$$

where $\alpha, \beta=1,2,3$, and $\delta_{\alpha \beta}$ is Kronecker's delta.

Poisson's equation (4) is an elliptic equation. Its solution depends on the boundary conditions. This equation is used - at each time step- to compute the source term $\nabla \phi$ which appears in Eqs (2) and (3). In multidimensional cases, Poisson's equation is solved by using the Fast Fourier Transform (FFT) algorithm (see Section 3.2.2). In 1D cases, Poisson's equation reduces to an ordinary differential equation and, consequently, the FFT is not necessary.

An equation of state $p=p(\rho, \epsilon)$ closes the system. The ideal gas equation of state $p=(\gamma-1) \rho \epsilon$ has been used in all applications in this paper. 
Let us focus on the evolutionary part of the above system. The evolution equations (5)-(7) can be written in the form:

$$
\frac{\partial \vec{u}}{\partial t}+\frac{\partial \vec{f}(\vec{u})}{\partial x}+\frac{\partial \vec{g}(\vec{u})}{\partial y}+\frac{\partial \vec{h}(\vec{u})}{\partial z}=\vec{s}(\vec{u})
$$

where $\vec{u}: \Re \times \Re^{3} \rightarrow \Re^{5}$ is the vector of unknowns:

$$
\vec{u}=\left[\delta, m_{x}, m_{y}, m_{z}, E\right]
$$

the three flux functions $\vec{F}^{\alpha} \equiv\{\vec{f}, \vec{g}, \vec{h}\}$ in the spatial directions $x, y, z$, respectively: $\Re^{5} \rightarrow \Re^{5}$, are defined by

$$
\begin{aligned}
& \vec{f}(\vec{u})=\left[\frac{m_{x}}{a}, \frac{m_{x}^{2}}{(\delta+1) a}+\frac{p}{a \rho_{B}}, \frac{m_{x} m_{y}}{(\delta+1) a}, \frac{m_{x} m_{z}}{(\delta+1) a}, \frac{(E+p) m_{x}}{a(\delta+1)}\right] \\
& \vec{g}(\vec{u})=\left[\frac{m_{y}}{a}, \frac{m_{x} m_{y}}{(\delta+1) a}, \frac{m_{y}^{2}}{(\delta+1) a}+\frac{p}{a \rho_{B}}, \frac{m_{y} m_{z}}{(\delta+1) a}, \frac{(E+p) m_{y}}{a(\delta+1)}\right] \\
& \vec{h}(\vec{u})=\left[\frac{m_{z}}{a}, \frac{m_{x} m_{z}}{(\delta+1) a}, \frac{m_{y} m_{z}}{(\delta+1) a}, \frac{m_{z}^{2}}{(\delta+1) a}+\frac{p}{a \rho_{B}}, \frac{(E+p) m_{z}}{a(\delta+1)}\right]
\end{aligned}
$$

and the sources $\vec{s}: \Re^{5} \rightarrow \Re^{5}$ are

$$
\begin{aligned}
\vec{s}(\vec{u})=[0 \quad,- & \frac{(\delta+1)}{a} \frac{\partial \phi}{\partial x}-H m_{x},-\frac{(\delta+1)}{a} \frac{\partial \phi}{\partial y}-H m_{y},-\frac{(\delta+1)}{a} \frac{\partial \phi}{\partial z}-H m_{z} \\
& \left.-\quad 3 H(E+p)-\frac{\rho_{B} H m^{2}}{(\delta+1)}-\frac{m_{x} \rho_{B}}{a} \frac{\partial \phi}{\partial x}-\frac{m_{y} \rho_{B}}{a} \frac{\partial \phi}{\partial y}-\frac{m_{z} \rho_{B}}{a} \frac{\partial \phi}{\partial z}\right]
\end{aligned}
$$

where $m_{x}=(\delta+1) v_{x}, m_{y}=(\delta+1) v_{y}$, and $m_{z}=(\delta+1) v_{z}$.

System (8) is a three-dimensional hyperbolic system of conservation laws with sources $\vec{s}(\vec{u})$. Let us introduce the Jacobian matrices associated to the fluxes:

$$
\mathcal{A}^{\alpha}=\frac{\partial \vec{F}^{\alpha}(\vec{u})}{\partial \vec{u}}
$$

hence, hyperbolicity demands that any real linear combination of the Jacobian matrices $\xi_{\alpha} \mathcal{A}^{\alpha}$ should be diagonalizable with real eigenvalues (LeVeque 1992). Knowledge of this 
fundamental property is not only of theoretical interest but is also of crucial importance from the numerical point of view (see below).

The spectral decompositions of the above Jacobian matrices in each direction, i.e., the eigenvalues and eigenvectors are explicitly written in the Appendix A. For Newtonian dynamics they can be found in, e.g., Glaister (1988). Let us point out that, in our case, the expansion factor does not influence the general features of the spectral decomposition derived in Newtonian dynamics.

The sources do not contain any term with differential operators acting on hydrodynamical variables $\vec{u}$. This is an important property consistent with the fact that the left hand side of Eq. (8) defines a hyperbolic system of conservation laws.

\subsection{Numerical case}

The main features of our multidimensional algorithm are the following:

1. It is written in conservation form. This is a very important property for a numerical algorithm designed to solve, numerically, a hyperbolic system of conservation laws. That is, in the absence of sources, those quantities that ought to be conserved -according to the differential equations- are exactly conserved in the difference form.

Let us comment how this fundamental idea is carried out in practice. The integral form of system (8) is

$$
\int_{\Omega} \frac{\partial \vec{u}}{\partial t} d \Omega+\int_{\Omega} \frac{\partial \vec{F}^{\alpha}}{\partial x^{\alpha}} d \Omega=\int_{\Omega} \vec{s} d \Omega
$$

where the domain $\Omega=[t, t+\Delta t] \times\left[x^{\alpha}, x^{\alpha}+\Delta x^{\alpha}\right] \forall \alpha$ defines a computational cell. Gauss' theorem allows us to write the above system in the following conservative 
form, well-adapted to numerical applications:

$$
\begin{aligned}
\left(\left\langle\vec{u}_{t+\Delta t}\right\rangle-\left\langle\vec{u}_{t}\right\rangle\right) \Delta V & =-\left(\int_{\Sigma_{x^{1}+\Delta x^{1}}} \widehat{\vec{f}} d t d x^{2} d x^{3}-\int_{\Sigma_{x^{1}}} \widehat{\vec{f}} d t d x^{2} d x^{3}\right) \\
& -\left(\int_{\Sigma_{x^{2}+\Delta x^{2}}} \widehat{\vec{g}} d t d x^{1} d x^{3}-\int_{\Sigma_{x^{2}}} \widehat{\vec{g}} d t d x^{1} d x^{3}\right) \\
& -\left(\int_{\Sigma_{x^{3}+\Delta x^{3}}} \widehat{\vec{h}} d t d x^{1} d x^{2}-\int_{\Sigma_{x^{3}}} \widehat{\vec{h}} d t d x^{1} d x^{2}\right)+\int_{\Omega} \vec{s} d \Omega
\end{aligned}
$$

where

$$
\begin{gathered}
\langle\vec{u}\rangle=\frac{1}{\Delta V} \int_{\Delta V} \vec{u} d x^{1} d x^{2} d x^{3} \\
\Delta V=\int_{x^{1}}^{x^{1}+\Delta x^{1}} \int_{x^{2}}^{x^{2}+\Delta x^{2}} \int_{x^{3}}^{x^{3}+\Delta x^{3}} d x^{1} d x^{2} d x^{3}
\end{gathered}
$$

Hence, the variation in time of quantities $\langle\vec{u}\rangle$ within $\Omega$-apart from the sources- is given by the fluxes $\vec{F}^{\alpha}$ on its boundary $\partial \Omega$.

The symbol ( $\wedge$ ) appearing on the fluxes on the right-hand side of (16) denote that they are calculated at the cell interfaces between the corresponding numerical cells by solving local Riemann problems (i.e., initial value problems with discontinuous data). The discretized version of (16) is obvious if we take into account that, at each time step $t=t^{n}$, data $\vec{u}_{i, j, k}^{n}$ are the cell-average of the variables $\vec{u}(x, y, z, t)$ :

$$
\vec{u}_{i, j, k}^{n}=\frac{\int_{x_{i-1 / 2}}^{x_{i+1 / 2}} \int_{y_{j-1 / 2}}^{y_{j+1 / 2}} \int_{z_{k-1 / 2}}^{z_{k+1 / 2}} \vec{u}\left(\mathrm{x}, \mathrm{y}, \mathrm{z}, \mathrm{t}^{\mathrm{n}}\right) \mathrm{dxdydz}}{\Delta x_{i} \Delta y_{j} \Delta z_{k}}
$$

where the set of indices $(i, j, k)$ stand for the $x, y, z$ directions, respectively, of a particular cell at a given time level. Indices $l-1 / 2$ and $l+1 / 2($ for $l=i, j, k)$ stand for the corresponding interfaces in each direction. These cell-averaged quantities are evolved in time (see below).

2. Reconstruction procedure. In order to increase spatial accuracy we have used two cell-reconstruction techniques. We have implemented a linear reconstruction, with the 
minmod function (Quilis et al. 1994) as a slope limiter. This limiter warranties that the method does not increase the total variation (TV), i.e.,

$$
T V\left(\vec{u}^{n+1}\right) \leq T V\left(\vec{u}^{n}\right)
$$

where the operation $T V$ is defined by

$$
T V\left(\vec{u}^{n}\right)=\sum_{\forall l}\left|\vec{u}_{l+1}^{n}-\vec{u}_{l}^{n}\right|
$$

and subindex $l$ stands for a generic spatial direction.

With this reconstruction our algorithm is a MUSCL-version (from monotonic upwind schemes for conservation laws, Van Leer 1979) and second order accurate in space. According to LeVeque (1992, page 184) MUSCL is a TVD (total variation diminishing) for scalar conservation laws.

We have also implemented a parabolic reconstruction (PPM) subroutine according to the procedure derived by Colella \& Woodward (1984). With the parabolic reconstruction the algorithm is third order accurate in space. Statements on the order of accuracy of an algorithm should be taken carefully when applied to a system and, moreover, when the numerical solution develops discontinuities. However, it can be proved that the above statements (in the sense of the local truncation error) are correct, at least for scalar equations.

Hence, from the cell-averaged quantities $\vec{u}_{i, j, k}^{n}$ we construct, in each direction, a piecewise linear or parabolic function which preserves monotonicity. Thus, the quantities, $\vec{u}_{i+\frac{1}{2}, j, k}^{R}, \vec{u}_{i, j+\frac{1}{2}, k}^{R}, \vec{u}_{i, j, k+\frac{1}{2}}^{R}$ and $\vec{u}_{i+\frac{1}{2}, j, k}^{L}, \vec{u}_{i, j+\frac{1}{2}, k}^{L}, \vec{u}_{i, j, k+\frac{1}{2}}^{L}$, can be computed; the superindices $R$ and $L$ stand for the values at the both sides of a given interface between neighbourhood cells. These values at each side of a given interface allow us to define the local Riemann problems. The numerical fluxes can be computed through the solution of these local Riemann problems. 
3. Numerical fluxes at interfaces. We have used a linearized Riemann solver following an approach similar to the one described by Roe (1981). The procedure, in each direction, starts by constructing the corresponding numerical flux according to Roe's prescription and in order to do that it is necessary to know the spectral decomposition of the Jacobian matrix $\mathcal{A}^{\alpha}$. Let us, for the sake of simplicity, focus on the $x$-direction. The numerical flux associated to the $x$-direction is:

$$
\widehat{\vec{f}}_{i+\frac{1}{2}, j, k}=\frac{1}{2}\left(\vec{f}\left(\vec{u}_{i+\frac{1}{2}, j, k}^{L}\right)+\vec{f}\left(\vec{u}_{i+\frac{1}{2}, j, k}^{R}\right)-\sum_{\eta=1}^{5}\left|\widetilde{\lambda}_{\eta}^{x}\right| \Delta \widetilde{\omega}_{\eta} \widetilde{\vec{R}}_{\eta}^{x}\right)
$$

where $\widetilde{\lambda}_{\eta}^{x}$ and $\widetilde{\vec{R}}_{\eta}^{x}(\eta=1,2,3,4,5)$ are, respectively, the eigenvalues (that is, the characteristic velocities) and the $\eta$-right eigenvector of the Jacobian matrix:

$$
\mathcal{A}_{\mathrm{i}+\frac{1}{2}, \mathrm{j}, \mathrm{k}}^{x}=\left(\frac{\partial \vec{f}(\vec{u})}{\partial \vec{u}}\right)_{\vec{u}=\left(\vec{u}_{i+\frac{1}{2}, j, k}^{L}+\vec{u}_{i+\frac{1}{2}, j, k}^{R}\right) / 2}
$$

calculated in the state which corresponds to the arithmetic mean of the states at each side of the interface. Quantities $\Delta \widetilde{\omega}_{\eta}$ - the jumps in the local characteristic variables through each interface- are obtained from the following relation

$$
\vec{u}^{R}-\vec{u}^{L}=\sum_{\eta=1}^{5} \Delta \widetilde{\omega}_{\eta} \widetilde{\vec{R}}_{\eta}^{x}
$$

where $\widetilde{\lambda}_{\eta}^{x}, \widetilde{\vec{R}}_{\eta}$ and $\Delta \widetilde{\omega}_{\eta}$, are functions of $\vec{u}$, which are calculated at each interface and, consequently, they depend on the particular values $\vec{u}^{L}$ and $\vec{u}^{R}$. Analogously, numerical fluxes in the $y$ direction, $\hat{\vec{g}}$, and $z$ direction, $\hat{\vec{h}}$, are obtained.

\section{Advancing in time:}

Once the numerical fluxes $\hat{\vec{f}}, \hat{\vec{g}}$, and $\hat{\vec{h}}$ are known, the evolution of quantities $\vec{u}_{i, j, k}$ is governed by

$$
\frac{d \vec{u}_{i, j, k}}{d t}=-\frac{\hat{\vec{f}}_{i+\frac{1}{2}, j, k}-\hat{\vec{f}}_{i-\frac{1}{2}, j, k}}{\Delta x_{i}}-\frac{\hat{\vec{g}}_{i, j+\frac{1}{2}, k}-\hat{\vec{g}}_{i, j-\frac{1}{2}, k}}{\Delta y_{j}}-\frac{\hat{\vec{h}}_{i, j, k+\frac{1}{2}}-\hat{\vec{h}}_{i, j, k-\frac{1}{2}}}{\Delta z_{k}}+\vec{s}_{i, j, k}
$$


An ordinary differential equation (ODE) solver derived by Shu and Osher (1988) has been used to solve Eq (25). It is a third order Runge-Kutta that does not increase the total variation of the numerical solution and preserves the conservation form of the scheme.

The standard Courant constraint on the time step reads as follows:

$$
\begin{aligned}
\left(\Delta t_{C}\right)_{i, j, k}=C F L_{1} \times \max & {\left[\frac{\Delta x_{i}}{\left|\lambda_{1}^{x}\left(x_{i+\frac{1}{2}, j, k}\right)-\lambda_{5}^{x}\left(x_{i-\frac{1}{2}, j, k}\right)\right|},\right.} \\
& \frac{\Delta y_{j}}{\left|\lambda_{1}^{y}\left(y_{i, j+\frac{1}{2}, k}\right)-\lambda_{5}^{y}\left(y_{i, j-\frac{1}{2}, k}\right)\right|}, \\
& \left.\frac{\Delta z_{k}}{\left|\lambda_{1}^{z}\left(z_{i, j, k+\frac{1}{2}}\right)-\lambda_{5}^{z}\left(z_{i, j, k-\frac{1}{2}}\right)\right|}\right]
\end{aligned}
$$

The value of the Courant time step, at each time level, is given by $\Delta t_{C}=\min \left(\Delta t_{C}\right)_{i, j, k}$, $\forall i, j, k$. Typical values of $C F L_{1}$ are running from 0.6 to 0.9 .

In pressureless tests, another constraint on the time step has been included. This is motivated by the fact that, in the absence of pressure, Courant condition does not guarantee the numerical stability of the system; hence, we introduce the dynamical time

$$
\Delta t_{d}=C F L_{2} \sqrt{\frac{3 \pi^{2}}{4 \rho}}
$$

where $\rho$ is the maximum density obtained in the previous time iteration. Typical values of the factor $C F L_{2}$ are of the order of $10^{-3}$. In any case, both Courant and dynamical time steps are computed and compared. The most restrictive of these time steps is used.

Particular attention must be paid to the source terms. The numerical treatment of the sources require the use of specific techniques, which depend on the desired accuracy and the complexity of these terms. In our case, the values of the quantities $\vec{s}_{i, j, k}$-at each cellcalculated at time level $t^{n}$ are added to the algorithm (25) for advancing in time.

For scalar equations and in the smooth part of the flow it can be shown that the linear reconstruction (our MUSCL-version) together with the Runge-Kutta procedure for 
advancing in time (see Appendix B) set up an algorithm which is second order accurate in space and third order accurate in time (in the sense of the local truncation error). When the PPM's subroutine is switched on our algorithm becomes globally third order accurate.

At this point several differences and analogies with the recent code published by Ryu et al. (1993) should be pointed out. It has been shown, at a purely theoretical level, that a MUSCL algorithm is TVD (see, e.g., LeVeque 1992). Numerical experiments carried out in 1D Newtonian dynamics (see, e.g., Yee 1989) show that MUSCL and the TVD scheme proposed by Harten (1983) - in which the code developed by Ryu et al. (1993) is based on - lead to similar results.

The most relevant differences with Ryu et al. (1993) code are the following:

1) Our code allows one to choose, by switching on an indicator, the reconstruction -linear or parabolic- according to the requirements between computational cost and accuracy in each particular application. As we have mentioned above, the parabolic reconstruction and our procedure for advancing in time makes our code globally third order unlike the second order of Ryu et al. (1993) code.

2) Our code uses a linearized Riemann solver due to Roe (1981) and when combined with the parabolic reconstruction allows to solve the Riemann problem with great accuracy.

3) Finally, let us discuss some important point concerning the so-called positively conservative property of a HRSC scheme. In a highly energetic flow -i.e., that one in which kinetic energy is much greater than the internal energy- it may happen that subtracting the kinetic energy -computed from a conservative numerical approximation for the conservation laws of mass and momentum- from conservative approximation for the conservation law for the total energy, could lead to a negative value for the internal energy, and, hence, to a failure of the numerical scheme (Einfeldt et al, 1991). Schemes such that both the internal 
energy and density remain positive throughout the computational process have been called positively conservative by Einfeldt et al. (1991). We do not know any theoretical analysis (theorem or lemma) proving that Harten's algorithm (Harten, 1983) -in which Ryu's code is based on- or MUSCL or PPM algorithms are positively conservative. In practice, we have no found in our numerical experiments (see below) any evidence of non-positiveness features; in particular, the spherical shock reflection (see below) test displays more than four orders of magnitude between kinetic energy and internal energy without any observed failure. Ryu et al. (1993) have overcome this question -the non-positiveness- by implementing in his code a bifurcation point that, during the evolution of the system, solves a different system of equations. In our code, if it were necessary, it is easy to implement a modified version of Roe's Riemann solver which - via the numerical fluxes- has the property of being a positively conservative algorithm (Einfeldt et al. 1991).

\section{Numerical tests}

In this section, our multidimensional code is tested. The most interesting aspects of each test are pointed out. Results are discussed.

In all the Figures considered in this Section we use marks (crosses, circles, squares and stars) to plot the numerical solutions obtained from our multidimensional code, while solid lines display the features of the exact or numerical solutions used for comparison.

\subsection{Tests involving shocks}

In the first test, we have focussed on standard shock tube problems whose analytical solutions are well known -in terms of the initial data- in studies of Newtonian (see, e.g., Yee 1989) or relativistic (see Martí \& Müller 1994) computational fluid dynamics. The 
second test involves a spherically symmetric shock. The exact solution can be found in Noh (1987). In these two tests, calculations have been carried out by switching off -in our codethe cosmological expansion and gravity terms, and pressure obeys the equation of state of an ideal gas with $\gamma=5 / 3$.

Both tests have been done with linear and parabolic reconstruction. In general PPM gives more accurate results. However, the price to pay is an increasing in the computational cost of parabolic reconstruction, which is greater than linear reconstruction by a factor of two or three.

\subsubsection{Shock tube}

The so-called shock tube problems are a set of solutions of different Riemann problems associated to the equations governing the dynamics of ideal gases. They involve, in general, the presence of shocks, rarefactions and contact discontinuities. Taking into account the fact that the analytical solution of the Riemann problem is well-known, they are considered as standard test-beds for checking a hydro-code.

In order to take advantage of the analytical solution of the standard shock tube problem, we have considered a computational domain defined by a cube (one unit length each edge) in which a discontinuity has been placed orthogonal to the cube diagonal joining the points $(0,0,0)$ to $(1,1,1)$. This discontinuity separates the system in two states. Initially, both states have the same density $(\rho=1)$ and velocity $(v=0)$. Pressure on the left (right) hand side of that discontinuity is $p=1(p=0.1)$. The grid used in these computations has $64 \times 64 \times 64$ cells. The evolution from the above initial data leads to the formation of a rarefaction wave, a contact discontinuity, and a shock travelling along the above mentioned diagonal. The location of the discontinuity has been chosen because it leads to 
nonvanishing fluxes in the three spatial directions, as occurs in the case of true 3D systems. Fig. 1 display the results obtained using linear (top panel) and parabolic (bottom panel) reconstruction. From these panels we can see an excellent agreement between the numerical and the analytical solutions. Let us point out that the shock is solved in only one cell in both cases. Numerical diffusion is slightly lesser with PPM, and the contact discontinuity is better solved with PPM as well.

\subsubsection{Shock reflection}

In the spherical shock reflection test, the integration domain is a cube initially $(t=0)$ filled by a uniform cold $(p=0)$ gas with density $\rho=1$. This gas moves towards the center of symmetry with a uniform radial velocity $v_{r}=-0.1$. At the central point, $r=0$, this velocity vanishes. Since the Mach number for the infalling gas is infinite (vanishing sound velocity), the resulting test is very severe. In Cartesian coordinates, the system can be considered as a true $3 \mathrm{D}$ one. The evolution of the above initial data leads to the formation -in the central region- of a strong reflecting shock which starts to propagate outwards. This shock leaves behind it a constant state of a thermalized and compressed gas at rest. The continuous lines of Fig. 2-3 correspond to the exact solution. As it can be seen in these Figures, the shock separates two well defined regions. More details about the exact solution and the shock reflection test can be found in Noh (1987).

In practice we have simulated the cold gas using a specific internal energy $\epsilon=10^{-6}$. Taken into account that in the preshock region velocity is $v_{r}=-0.1$, the ratio between the thermal energy $\left(E_{t h}\right)$ and the total energy $(\mathrm{E})$ is $\frac{E_{t h}}{E} \geq 10^{-4}$. We want to point out that even with this strong condition the results have been acceptable, and the code seems to support ratios between thermal and total energy up to four orders of magnitude. 
In the numerical experiments we discuss in this subsection the center of symmetry is placed at the center of a cube having an edge of 2 units length. This spatial computational domain has been partitioned into a uniform grid of $N \times N \times N$ cells. Two values for $N$ have been considered $(N=41,81)$.

Figs. 2 and 3 show results obtained by using linear and parabolic reconstruction, respectively. They have been selected at $t=12$.

The top (bottom) panel of Fig. 2 displays the values of the radial velocity (density) on the diagonal joining the points $(-1,-1,-1)$ and $(1,1,1)$, namely, on the main diagonal. Square and star (circle and cross) marks correspond to the use of $41 \times 41 \times 41(81 \times 81 \times 81)$ cells. The squares (circles) give the numerical values of $v_{r}$ and $\rho$ at some points of the half-diagonal joining the points $(0,0,0)$ and $(1,1,1)$, while the stars (crosses) correspond to the symmetric points with respect to the origin, which lie on the half-diagonal joining the points $(-1,-1,-1)$ and $(0,0,0)$. Let us point out that for any pair of these symmetric points, the resulting values of $v_{r}$ and $\rho$ are indistinguishable, their relative differences being smaller than $2 \times 10^{-5}$. It can be concluded that, for any chosen direction, the symmetry with respect to the origin is numerically preserved by the code. A visual inspection of Fig. 2 shows that: (1) the shock appears located at the position $r=r_{\text {shock }}$ given by the exact solution (continuous line), (2) it is sharply solved in two or three cells, and (3) the most important errors appear in the region $r<r_{\text {shock}}$. It is remarkable that these errors decrease as the number of cells increases (compare circle with square marks); this means that the numerical solution has a good convergence rate.

In the case of the experiment involving $81 \times 81 \times 81$ cells, some quantities describing the deviations with respect to the exact solution have been estimated. Leaving aside the points defining the shock, the maximum relative error of density is $19 \%$ and the mean relative error is $10 \%$. The maximum absolute error of radial velocity is $-6.8 \times 10^{-3}$ and 
the mean absolute error is $-1.06 \times 10^{-3}$. The maximum errors appear only in a few points located near $r=0$ and $r=r_{\text {shock}}$. Taking into account that we are using very coarse grids, our results are encouraging. As it should happen, the errors decrease as the number of cells increases.

Fig. 3 is analogous to Fig. 2 but using parabolic reconstruction. The numerical solution obtained with PPM exhibits also the fundamental property of being symmetric in the sense explained before. Shock is solved in a numerical cell. The constant post-shock state in density is reached by PPM with a resolution better than MUSCL. In particular, for the case of poor grids $(N=41)$ the differences between MUSCL and PPM are more outstanding.

Figs. 4 and 5 display the results obtained with MUSCL at $t=15$.

Fig. 4 shows three slices of the spatial computational domain. The top, intermediate and bottom panels correspond to the coordinates $z=0.875, z=0$ and $z=-0.875$, respectively. Left (right) panel refers to densities (velocities). The top and bottom panels of Fig. 3 show that (i) the boundary conditions on the cube faces produce slight deviations with respect to the spherical symmetry (dark bands near the faces), (ii) except for these small deviations, numerical solutions show a strong spherical symmetry, (iii) the slices $z=0.875$ and $z=-0.875$ are indistinguishable, as it is expected on account of the intrinsic features of the code, and (iv) the shock has not arrived at these regions. Finally, the intermediate panel shows a spherically symmetric strong shock. The region $r<r_{\text {shock }}$ appears to be quasi-homogeneous. It only involves some small fluctuations. The greatest deviations from the exact solution -which is homogeneous in this region- appear along those directions parallel to the axis, which are contained inside the corresponding coordinate plane. These effects are related to the boundary conditions.

Fig. 5 is presented essentially to describe the pressure behavior. The function 
$p=p(t=15, x, y, z=0)$ is displayed. Let us pay attention to the following features: The cylindrical structure of the surface (whose height is the pressure jump at the shock), the sharp structure of the strong shock, and the fact that boundary conditions lead to maximum errors in the directions described above.

In our opinion, this test is so severe and the results good enough to suggest that the use of a finer grid -with the corresponding consumption of machine resources (RAM memory and CPU)- would allow to improve definitively the numerical solution.

In the above numerical experiments, CPU cost - in a HP Apollo 9000/712 - is 0.25 ms per timestep and per numerical cell.

\subsection{Cosmological tests}

The third test in this paper is based on the Zel'dovich 1D solution, which is an exact solution of the equations describing cosmological pressureless inhomogeneities. This case includes cosmological expansion, but it is not a 3D case.

The fourth test uses a numerical planar 1D solution of the evolution equations describing a cosmological inhomogeneity with pressure. This solution was obtained by Quilis et al. (1994) using a 1D code.

The fifth (sixth) test is based on an analytical (numerical) spherically symmetric solution of the equations describing the evolution of cosmological Newtonian inhomogeneities without (with) pressure. The analytical solution of the fifth test is described in Peebles (1980) and the numerical solution of the sixth test was obtained by Quilis et al. (1995). These solutions can be considered as 1D ones in spherical coordinates; nevertheless, in Cartesian coordinates, they can be treated as fully 3D solutions including cosmological expansion and gravity. 
All the cosmological tests have been done with the linear reconstruction.

\subsubsection{Cosmological tests with planar symmetry}

In spite of the fact that our code is multidimensional, any 1D solution can be used in order to perform a significant test; in fact, each of these solutions can be used to set particular initial conditions for running our multidimensional code. After integration (at arbitrary time), this code must maintain the planar symmetry and reproduce the chosen planar solution. In planar cases, Poisson's equation reduces to an ordinary differential one, which is solved by using an ODE solver; hence, tests based on planar solutions do not give any information about the behavior of the method used to solve Poisson's equation (FFT) in the general case.

The $1 D$ Zel'dovich solution is an exact solution of the hydrodynamic equations in the planar case (Shandarin \& Zel'dovich 1989). High density contrasts are compatible with this solution, which is valid before caustic formation. Multidimensional Zel'dovich's solution is only an approximate one. This solution is only valid in the mildly nonlinear regime (density contrast up to a few units). From these comments about the features of Zel'dovich's solution, it follows that only the 1D version of this solution is appropriate in order to test a numerical code in the strongly nonlinear regime.

The first planar cosmological test is based on the 1D Zel'dovich solution. Initial conditions are given at redshift $Z=50$. As it is well known, Zel'dovich's solution is completely defined by the potential of the velocity field, $\phi_{z}(q)$, which is assumed to have the following form: $\phi_{z}(q)=-A \cos k q, q$ being a Lagrangian coordinate, and $\mathrm{A}$ and $\mathrm{k}$ two free parameters. In this paper, the values of these parameters are: $A=2.7 \times 10^{-6} h M p c^{-1}$ and $k=35.2$ (see Quilis et al. 1994). The top panels of Fig. 6 show the density contrast 
(right) and velocity (left) profiles at time $t / t_{c}=0.94, t_{c}$ being the critical time of caustic formation for the chosen inhomogeneity $\left(t_{c}=1.1 \times t_{0}\right)$.

The results are quite good. Numerical and analytical solutions appear to be comparable. The mean relative errors in velocity and density are of the order of $1 \%$, except at the maximum (central cell), where the relative error in the density contrast is the greatest $(\sim 30 \%)$.

The second planar cosmological test is based on a numerical $1 D$ solution obtained by Quilis et al. (1994); in this case, pressure obeys the equation of state $p=(\gamma-1) \epsilon \rho$, with $\gamma=5 / 3$; the initial value of $\epsilon$ is $10^{-6}$. Initial conditions are given at redshift $Z=50$. The initial profiles are the same as those in the first cosmological planar test (see above). In the bottom panels of Fig. 6, the density contrast (right) and the velocity (left) profiles are shown at time $t / t_{0}=1.0$. The solid line corresponds to the $1 \mathrm{D}$ solution of Quilis et

al. (1994). In the presence of pressure, the spatial gradients are smaller than those of the corresponding pressureless case; for this reason, the agreement between the two solutions compared in this test, is greater than that of the previous planar cosmological test. This fact is illustrated by Fig. 6, where it can be seen that, in the presence of pressure (bottom panel), the differences between the compared solutions are smaller than in the pressureless case (top panel).

In both planar cosmological tests, the spatial grid has 200 cells in the relevant direction.

\subsubsection{Cosmological tests with spherical symmetry}

These spherical cosmological tests have two important features: The symmetry does not reduce the number of relevant directions in Cartesian coordinates, and the general 3D Poisson equation is solved by using the FFT (Press et al. 1987). These numerical 
experiments complement previous ones because they are multidimensional tests including gravity and expansion.

In the multidimensional general case, our code solves Poisson's equation for the gravitational potential -at each time step- by using the FFT. The use of this technique requires periodic boundary conditions on the faces of an auxiliary cube (the elemental cube of the FFT). This fact must be carefully taken into account in order to interpret our results.

The FFT is used as follows: The density contrast in physical space -with suitable boundary conditions- is the starting point. We can distinguish three consecutive steps: (1) A FFT gives $\delta_{\vec{k}}$ (the Fourier component of $\delta$ ), (2) Poisson's equation in Fourier space, $\phi_{\vec{k}}=-\delta_{\vec{k}} / k^{2}$, is used in order to get $\phi_{\vec{k}}$, and (3) the inverse FFT leads to the required gravitational potential in physical space.

The first test of this section uses an exact spherically symmetric solution of Eqs. (1)-(4) (Peebles 1980). Initial conditions are given at redshift $Z=7$. The initial profile of the density contrast is

$$
\delta_{i}(r)=\frac{\delta_{c}}{1+\left(\frac{r}{r_{v}}\right)^{1.8}}
$$

with $r=\sqrt{x^{2}+y^{2}+z^{2}}, \delta_{c}$ being the amplitude of the density contrast and $r_{v}$ the value of $r$ there where $\delta$ reduces to one-half of $\delta_{c}$. The initial peculiar velocity corresponds to vanishing nongrowing modes and it can be obtained from the initial density contrast. These initial profiles are used as inputs in our code. In the applications of this paper, the free parameters of the density profile are assumed to be $r_{v}=0.6 h^{-1} M p c$ and $\delta_{c}=0.26$. These values were also used in Quilis et al. (1995) in order to simulate a rich Abell cluster at redshift $Z=0.02$.

Although a spherical solution is used in order to give initial conditions, our numerical code cannot reproduce this solution in all the elemental cubes; in order to understand this fact and interpret the results, it should be taken into account that the FFT gives the 
gravitational field produced by an ideal distribution of elemental cubes filling the space (not by a spherically symmetric distribution of matter filling all the space). Hence, the gravitational field is comparable to that of the spherical solution in the central region of the cube, but not far from the center of symmetry, where the contribution to the field of the neighbouring cubes is relevant.

From the important considerations above, it follows that: (i) our code should reproduce the exact spherical solution in the central part of the elemental cube and (ii) our numerical results should deviate from those of the exact spherical solution as the distance to the symmetry center increases. This separation should also appear in the absence of pure numerical errors.

In this test, two grids having $32 \times 32 \times 32$ and $64 \times 64 \times 64$ cells are used and the results are compared. The top panel of Fig. 7 displays the density contrast (right) and the peculiar velocity (left) at time $t / t_{0}=0.89$. Solid line corresponds to the exact spherical solution. Circles (crosses) stand for the numerical results obtained with the $32 \times 32 \times 32$ $(64 \times 64 \times 64)$ grid. In the central region of the box, the relative difference between numerical and analytical values of $v$ and $\delta$ are smaller than $3 \%$ for the $32 \times 32 \times 32$ grid and smaller than $1 \%$ for the $64 \times 64 \times 64$ grid (except for the central point). As expected, for both grids, the relative differences increase as the distance to the center increases, but they are smaller than $10 \%$ everywhere. This difference is not merely a numerical error but the superposition of a numerical error and the deviation of two solutions coming from two different problems: an ideal distribution of elemental cubes filling the space and a spherically symmetric distribution of matter filling all the space. Let us remark that satisfactory results have been obtained with the extremely coarse grid of $32 \times 32 \times 32$ cells.

The second test of this section is based on a numerical spherically symmetric solution derived by Quilis et al. (1995). In this case, the initial density profile has the form (28) 
and the values of the parameters $r_{v}$ and $\delta_{c}$ are the same as in the first spherical test. The initial value of $\epsilon$ is $5 \times 10^{-6}$. The equation of state is $p=(\gamma-1) \epsilon \rho$, with $\gamma=5 / 3$. A grid of $64 \times 64 \times 64$ cells has been used.

The bottom panel of Fig. 7 shows the density profile (right) and the peculiar velocity (left) at time $t / t_{0}=0.89$. The continuous line corresponds to the numerical solution with pressure due to Quilis et al. (1995). Although the initial profiles and the final time are the same as in the first spherical test, the density contrast has reached central values smaller than in that test. This effect is produced by the spatial pressure gradients. Apart from this difference, both spherical tests display comparable features (see Fig. 7). Velocities show the same behavior as in the previous test.

In the above numerical calculations, CPU cost - in a HP Apollo 9000/712 - is 0.36 ms per timestep and per numerical cell.

\section{General discussion}

In this paper, we have numerically solved the full multidimensional system of hydrodynamical equations describing the evolution of a gas -including gravity and an

expanding cosmological background-taking advantage of the fact that they are a system of conservation laws with sources. This property is crucial for using modern HRSC techniques.

A multidimensional hydrodynamic code based on these techniques has been built up. This code includes the possibility of choosing between two spatial reconstructions in order to get better resolutions, i.e. a linear reconstruction (MUSCL) or a parabolic reconstruction (PPM).

An algorithm for solving Poisson's equation at each time step is included as well. This Poisson solver is based on the FFT. The use of this transform is appropriated because the 
code described in Section. 2 gives the density at each time step and this density is the only ingredient required by the FFT in order to compute the gravitational potential; in other words, if the FFT is used, no unknown boundary conditions for the gravitational potential are required as inputs. Finally, the time elapsed by the FFT increases very slowly as the number of points per edge - of the elemental cube- increases. Although the use of the FFT has important advantages, this technique only leads to admissible simulations in the central part of the elemental box. This is an unavoidable limitation attached to the use of the FFT. The elemental cube must be carefully chosen in each case.

Our code has passed successfully a battery of six severe tests. Four of them (shock tube problem, spherical shock reflection, Zel'dovich's solution and the Newtonian pressureless spherically symmetric solution) are in fact considered as standard bed-tests in classical and cosmological hydrodynamics. We have implemented two more numerical tests from previous numerical solutions.

The behaviour of our code is good in all six cases. The tests show that the code works in the presence of shocks, rarefactions, contact discontinuities, cosmological expansion, gravity and pressure. In the spherical cosmological test, it has been seen that high density contrasts of the order of $10^{2}$ can be reached by using spatial grids with $64 \times 64 \times 64$ points (see Fig. 7). Larger density contrasts would require a greater number of points and, consequently, they would have a greater computational cost. Grids having $128 \times 128 \times 128$ nodes should allow the description of the hot gas component located inside clusters up to density contrasts between $10^{2}$ and $10^{3}$ (rich clusters).

In cosmological applications and as Ryu et al. (1993) pointed out, it might happen that regions having large kinetical -compared with the thermal energy-appear. As we have discussed before, a conservative algorithm could lead to important numerical difficulties. In practice we have not noticed this problem in any of the tests presented in this paper, overall 
in the shock reflexion test where $\frac{E_{t h}}{E} \geq 10^{-4}$ and the analytical solution was recovered quite well.

Important perspectives arise in the case of several cosmological problems, specially, if the code presented in this paper is coupled to a N-body one (see Bertschinger \& Gelb 1991 for a description of this kind of codes) describing the evolution of the pressureless matter. In order to design this coupling, it should be taken into account that the hot gas and the pressureless component are gravitationally coupled. Let us point out that the resulting coupled code could lead to very realistic simulations of rich clusters, in which, the observed features of the baryonic component would play an important role. See Quilis et al.(1995) for a discussion about this point.

This work has been supported by the Conselleria d'Educació i Ciència de la Generalitat Valenciana (grant GV-2207/94) and the Spanish DGICYT (grant PB94-0973). Dr. M. Steinmetz and an anonymous referee are acknowledged for their valuable comments and criticism. Authors have enjoyed fruitful conversations with J.Mㅁ․ Martí, J.A. Miralles, V. Romero, J.V. Arnau and F. Banyuls. Calculations were carried out in a IBM 30-9021 VF at the Center de Informàtica de la Universitat de València, and in a HP Apollo 712. V.Quilis thanks to the Conselleria d'Educació i Ciència de la Generalitat Valenciana for a fellowship.

\section{A. Spectral decompositions for the Jacobian matrices}

The hydrodynamic equations (5)-(7) can be written in the form of Eq. (8), where the vector of unknowns $\vec{u}$ is given by Eq. (9), and the fluxes $[\vec{f}, \vec{g}, \vec{h}]$ in the directions $x, y, z$ respectively, and sources $\vec{s}$, are given by Eqs. (10)-(13).

If the Jacobian matrices in each direction, $\mathcal{A}^{\S}=\frac{\partial \overrightarrow{\{}(\vec{\Pi})}{\partial \vec{\Pi}}, \mathcal{A}^{\dagger}=\frac{\partial \vec{\jmath}(\vec{\Pi})}{\partial \vec{\Pi}}$, and $\mathcal{A}^{\ddagger}=\frac{\partial \overrightarrow{\langle}(\vec{\Pi})}{\partial \vec{\Pi}}$, have 
real eigenvalues and the right eigenvectors form a complete set then the system (8) is called an hyperbolic system of conservation laws with sources.

The spectral decomposition, i.e. eigenvalues, right and left eigenvectors, are needed in order to build the numerical code. For sake of simplicity we list here, the eigenvalues, and the right and left eigenvectors for $\mathcal{A}^{\S}$. The spectral decompositions in the other directions are formally identical.

Eigenvalues:

$$
\begin{aligned}
\lambda_{1}^{x} & =\frac{v_{x}+c_{s}}{a} \\
\lambda_{2}^{x}=\lambda_{3}^{x}=\lambda_{4}^{x} & =\frac{v_{x}}{a} \\
\lambda_{5}^{x} & =\frac{v_{x}-c_{s}}{a}
\end{aligned}
$$

where $c_{s}$ is the sound speed $\left(c_{s}=(\gamma p / \rho)^{1 / 2}\right)$.

Right eigenvectors:

$$
\begin{gathered}
\vec{R}_{1}^{x}=\left(\begin{array}{c}
1 \\
v_{x}+c_{s} \\
v_{y} \\
v_{z} \\
\frac{p+E}{\rho}+v_{x} c_{s}
\end{array}\right) \quad, \quad \vec{R}_{2}^{x}=\left(\begin{array}{c}
1 \\
v_{x} \\
v_{y} \\
v_{z} \\
\frac{v^{2}}{2}
\end{array}\right), \vec{R}_{3}^{x}=\left(\begin{array}{c}
0 \\
0 \\
1 \\
0 \\
v_{y}
\end{array}\right), \\
\vec{R}_{4}^{x}=\left(\begin{array}{c}
0 \\
0 \\
0 \\
1 \\
v_{z}
\end{array}\right), \vec{R}_{5}^{x}=\left(\begin{array}{c}
v_{x}-c_{s} \\
v_{y} \\
v_{z} \\
\frac{p+E}{\rho}-v_{x} c_{s}
\end{array}\right)
\end{gathered}
$$

being $v^{2}=v_{x}^{2}+v_{y}^{2}+v_{z}^{2}$. 
Left Eigenvectors: Orthonormal to the right vectors, $L_{m} \cdot R_{l}=\delta_{m l}$,

$$
\begin{gathered}
\vec{L}_{1}^{x}=\left[\frac{v^{2}(\gamma-1)-2 v_{x} c_{s}}{4 c_{s}^{2}}, \frac{c_{s}-v_{x}(\gamma-1)}{2 c_{s}^{2}} \quad, \quad-\frac{v_{y}(\gamma-1)}{2 c_{s}^{2}},\right. \\
\left.-\frac{v_{z}(\gamma-1)}{2 c_{s}^{2}}, \frac{\gamma-1}{2 c_{s}^{2}}\right] \\
\vec{L}_{2}^{x}=\left[1-\frac{(\gamma-1) v^{2}}{2 c_{s}^{2}}, \frac{v_{x}(\gamma-1)}{c_{s}^{2}}, \frac{v_{y}(\gamma-1)}{c_{s}^{2}}, \frac{v_{z}(\gamma-1)}{c_{2}^{2}},-\frac{\gamma-1}{c_{s}^{2}}\right] \\
\vec{L}_{3}^{x}=\left[-v_{y}, 0,1,0,0\right] \\
\vec{L}_{4}^{x}=\left[-v_{z}, 0,0,1,0\right] \\
\vec{L}_{5}^{x}=\left[\frac{v^{2}(\gamma-1)+2 v_{x} c_{s}}{4 c_{s}^{2}},-\frac{c_{s}+v_{x}(\gamma-1)}{2 c_{s}^{2}} \quad, \quad-\frac{v_{y}(\gamma-1)}{2 c_{s}^{2}},\right. \\
\left.-\frac{v_{z}(\gamma-1)}{2 c_{s}^{2}}, \frac{\gamma-1}{2 c_{s}^{2}}\right]
\end{gathered}
$$

\section{B. Summary of the algorithm}

Here, we summarize the steps in order to build up our hydro-code. In one of them, the spectral decompositions of the Jacobian matrices described in the Appendix A, are needed. For more details see Section 2.

The scheme for the numerical procedure in updating vector $\vec{u}^{n}$ to $\vec{u}^{n+1}$, is the following:

1. The unknowns $\vec{u}$ are known at the center of the numerical cells at the time step $n$, i.e. $\vec{u}^{n}$.

2. Reconstruction procedure allows to compute the unknowns at the interface between a cell and its neighbours. It must be done for each direction. For instance, in the $x$ direction is

$$
\vec{u}_{i-1, j, k}^{n}, \vec{u}_{i, j, k}^{n}, \vec{u}_{i+1, j, k}^{n} \Longrightarrow\left\{\begin{array}{c}
\vec{u}_{i+\frac{1}{2}, j, k}^{R} \\
\vec{u}_{i+\frac{1}{2}, j, k}^{L}
\end{array}\right.
$$


Reconstruction, in our code, can be linear or parabolic (PPM).

3. Numerical fluxes are computed using Roe prescription, Eq.(22). For example in the $x$ direction,

$$
\widehat{\vec{f}}_{i+\frac{1}{2}, j, k}^{n}=\frac{1}{2}\left(\left(\vec{f}\left(\vec{u}_{i+\frac{1}{2}, j, k}^{L}\right)^{n}+\vec{f}\left(\vec{u}_{i+\frac{1}{2}, j, k}^{R}\right)^{n}-\sum_{\eta=1}^{5}\left|\tilde{\lambda}_{\eta}^{x}\right| \Delta \widetilde{\omega}_{\eta} \widetilde{\vec{R}}_{\eta}^{x}\right)\right)
$$

where

$$
\vec{L}_{\eta}^{x} \cdot\left(\vec{u}_{i+\frac{1}{2}, j, k}^{R}-\vec{u}_{i+\frac{1}{2}, j, k}^{L}\right)=\Delta \widetilde{\omega}_{\eta} \quad, \eta=1, \ldots, 5
$$

symbol $(\sim)$ refers to mean values in the interface. The fluxes in directions $y, z, \vec{g}$ and $\vec{h}$, are obtained analogously.

4. Poisson's equation is solved by using FFT.

5. Sources are obtained at each cell, $\vec{s}\left(\vec{u}_{i, j, k}\right)$.

6. Advancing in time,

$$
\vec{u}_{i, j, k}^{n+1}=\vec{u}_{i, j, k}^{n}-\Delta t \mathcal{L}\left(\vec{\Pi}_{\rangle, 1, \|}^{\backslash}\right)
$$

where $\Delta t=t^{n+1}-t^{n}$, and $\mathcal{L}$ is the operator

$$
\begin{aligned}
\mathcal{L}\left(\vec{\Pi}_{\rangle,|,| \|}\right) & =\frac{\hat{\vec{f}}\left(u_{i+\frac{1}{2}, j, k}\right)-\hat{\vec{f}}\left(u_{i-\frac{1}{2}, j, k}\right)}{\Delta x_{i}}+\frac{\hat{\vec{g}}\left(u_{i, j+\frac{1}{2}, k}\right)-\hat{\vec{g}}\left(u_{i, j-\frac{1}{2}, k}\right)}{\Delta y_{j}} \\
& +\frac{\hat{\vec{h}}\left(u_{\left.i, j, k+\frac{1}{2}\right)}-\hat{\vec{h}}\left(u_{i, j, k-\frac{1}{2}}\right)\right.}{\Delta z_{k}}+\vec{s}\left(u_{i, j, k}\right)
\end{aligned}
$$

A third order Runge-Kutta, proposed by Shu and Osher (1988), has been chosen in order to solve Eq. (B4). The expressions corresponding to this Runge-Kutta like solver are:

$$
\vec{u}^{1}=\vec{u}^{n}+\Delta t \mathcal{L}(\vec{\Pi} \backslash)
$$




$$
\begin{gathered}
\vec{u}^{2}=\frac{3}{4} \vec{u}^{n}+\frac{1}{4} \vec{u}^{1}+\frac{1}{4} \Delta t \mathcal{L}\left(\vec{\Pi}^{\infty}\right) \\
\vec{u}^{n+1}=\frac{1}{3} \vec{u}^{n}+\frac{2}{3} \vec{u}^{2}+\frac{2}{3} \Delta t \mathcal{L}\left(\vec{\Pi}^{\in}\right)
\end{gathered}
$$

and $\vec{u}^{1}, \vec{u}^{2}$ being two intermediate states.

7. The unknowns $\vec{u}$ are known at the center of the numerical cells at the time step $n+1$, i.e. $\vec{u}^{n+1}$. 


\section{REFERENCES}

Bertschinger E., 1991, in Lectures Notes in Physics, New insights into the Universe, eds. Martínez V.J., Portilla M. \& Sáez D., Springer-Verlag, Valencia, p. 65

Bertschinger E. \& Gelb, J.M., 1991, Comp. in Phys, 5, 164

Cen R., 1992, ApJ. Suppl., 78, 341

Colella P. \& Woodward P.R., 1984, J. Comp. Phys., 54, 174

Einfeldt B., Munz C.D., Roe P.L. \& Sjögreen B., 1991, J. Comp. Phys., 92, 273

Gingold R.A. \& Monaghan J.J., 1977, MNRAS, 181, 375

Glaister P., 1988, J. Comp. Phys., 77, 361

Gurvatov S., Saichev A. \& Shandarin S.F., 1989, MNRAS, 236, 385

Lax P., 1973, in "Regional Conference Series Lectures in Applied Math.". SIAM, Philadelphia

LeVeque R.J., 1992, Numerical Methods for Conservation Laws, Birkhäuser, p. 206

Lucy L.B., 1977, Astron. J., 82, 1013

Martí J.M므. \& Müller E., 1994, J.Fluid Mech, 258, 317

Martí J.Ma․, Müller E., Font J.A. \& Ibáñez J.Mํa ., 1995, ApJ. Lett., in press.

Matarrese S., Lucchin F., Moscardini L. \& Sáez D., 1992, MNRAS, 259, 437

Mellema G., Eulderink F. \& Icke V., 1991, A\&A, 252, 718

Müller E., 1994, in Supernovae, ed. by Bludman S.A., Moschkowitch R. \& Zinn-Justin J., North-Holland 
Noh W.F., 1987, J. Comp. Phys., 72,78

Peebles, P.J.E., 1980, The Large Scale Structure of the Universe. Princenton University Press

Press H., Flannery B.P., Teukolsky S.A. \& Vetterling W.T., 1987, Numerical Recipies. The art of Scientific Computing. Cambridge University Press

Quilis V., Ibáñez J.Ma․ \& Sáez D., 1994, A\&A, 286, 1

Quilis V., Ibáñez J.Ma․ \& Sáez D., 1995, MNRAS, 277, 445

Roe P.L., 1981, J. Comp. Phys., 43, 357

Ryu D., Ostriker J.P., Kang H. \& Cen R., 1993, ApJ, 414, 1

Shandarin S.F. \& Zel'dovich Ya.B., 1989, Rev. Mod. Phys., 61, 185

Shu C. \& Osher C., 1988, J. Comp. Phys., 77, 439

Sunyaev R.A. \& Zel'dovich Ya. B., 1980, A\&A, 18, 537

Van Leer B., 1979, J. Comp. Phys., 32, 101

Yee H.C., 1989, in VKI Lecture Notes in Computational Fluid Dynamics, von Karman Institute for Fluid Dynamics, Belgium

Zel'dovich Ya.B., 1970, A\&A, 5, 84 
Fig. 1.- Plot of the analytical (solid line) and two numerical (crosses) solutions for a shock tube numerical experiment. Top and bottom panel show results using linear (MUSCL) and parabolic (PPM) reconstruction, respectively. In both panels, left central and right figures display density $\rho$, pressure $p$ and velocity $v$, respectively, as functions of the parameter $l=\left(x^{2}+y^{2}+z^{2}\right)^{1 / 2}$.

Fig. 2.- Plot of the analytical (solid line) and the numerical (marks) solutions for two reflection shock experiments at $t=12$ using linear reconstruction (MUSCL). Top (bottom) panel corresponds to radial velocity (density). Squares (stars) represent points of the halfdiagonal joining the points $(0,0,0)$ and $(1,1,1)((-1,-1,-1)$ and $(0,0,0))$ in the experiment involving $41 \times 41 \times 41$ cells. Circles and crosses play the role of squares and stars, respectively, in the case of the experiment using $81 \times 81 \times 81$ cells.

Fig. 3.- Analogous to Fig. 2 but using parabolic reconstruction (PPM).

Fig. 4. - Left (right) panels show density (radial velocity) at $t=15$. Top, intermediate and bottom panels correspond to $z=0.875, z=0$, and $z=-0.875$, respectively. At the left side of each panel, it is placed a palette displaying the grey scale.

Fig. 5.- Plot of the function $p=p(t, x, y, z)$ for $z=0$ and $t=15$.

Fig. 6. - Left (right) top panel shows the peculiar velocity (density contrast) for the Zel'dovich solution (solid line) and the numerical one (crosses) at time $t / t_{0}=1$. Similar for the bottom panels, where the solid line corresponds to a numerical solution due to Quilis et al. (1994).

Fig. 7.- Left (right) top panel shows the peculiar velocity (density contrast) for a pressureless spherical solution (solid line) and the numerical one (circles for $32 \times 32 \times 32$ and crosses for $64 \times 64 \times 64$ cells) at time $t / t_{0}=0.89$. Similar for the bottom panels, where 
the solid line corresponds to a numerical spherically symmetric solution due to Quilis et al. (1995). 

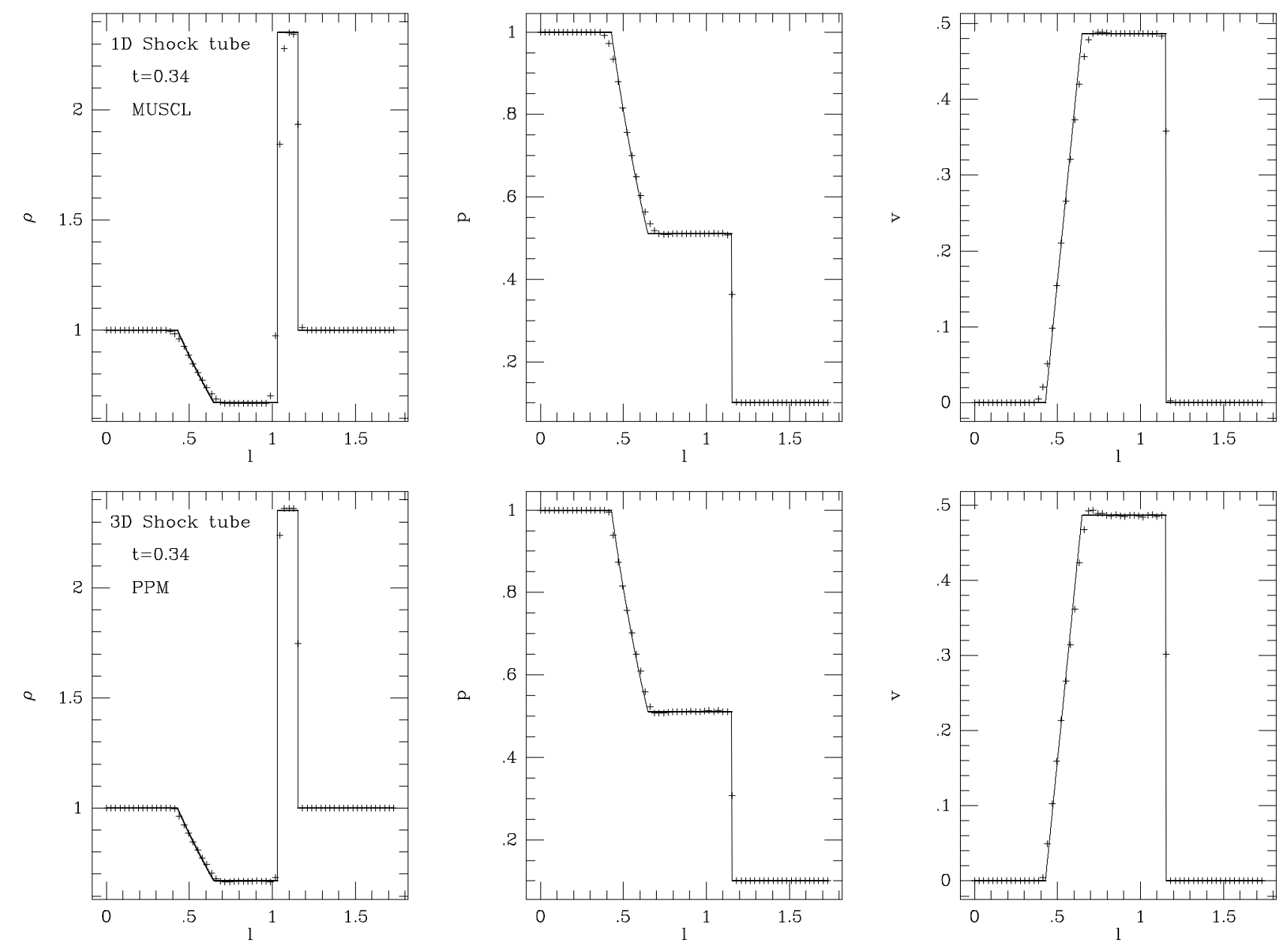

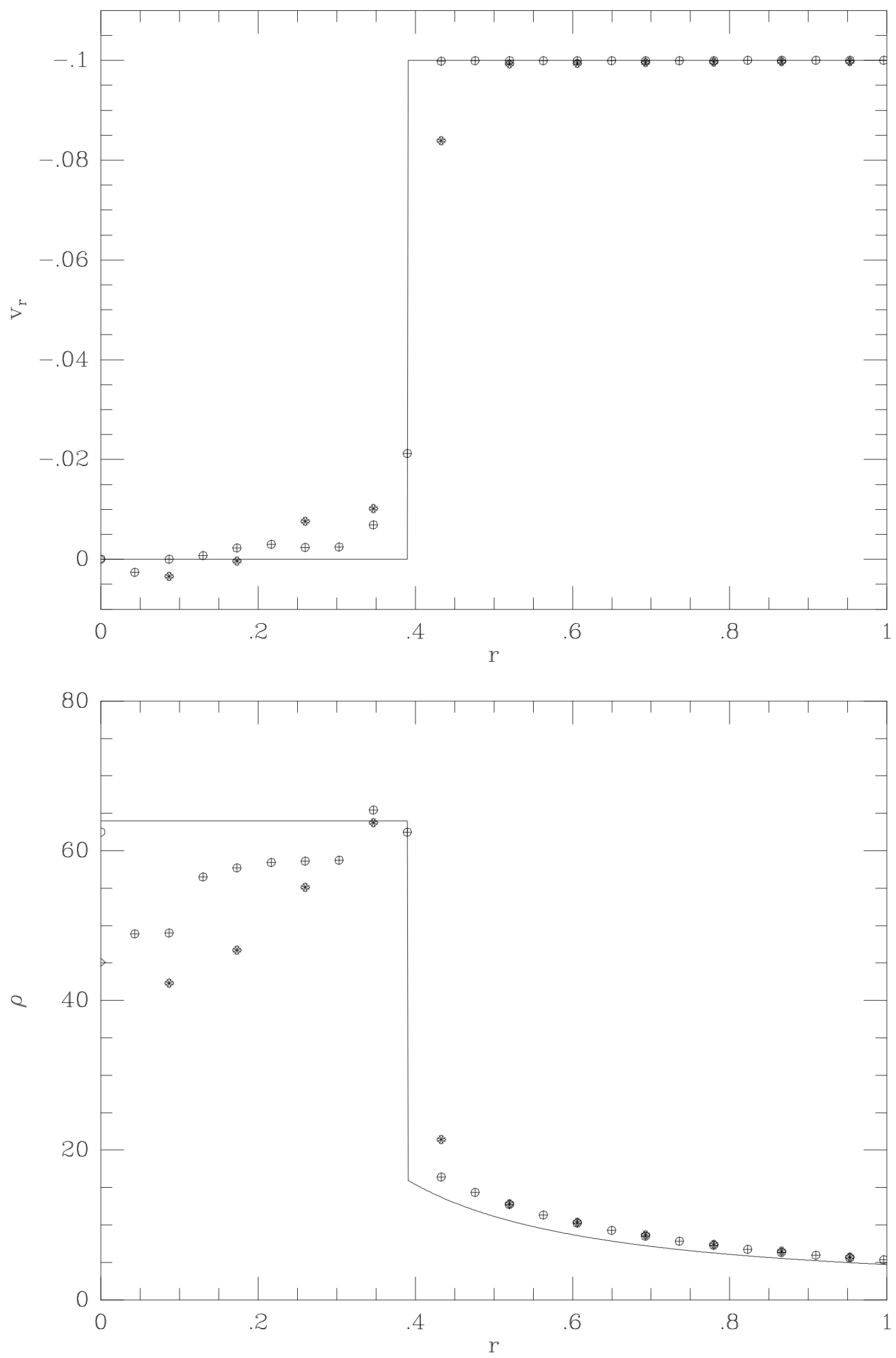

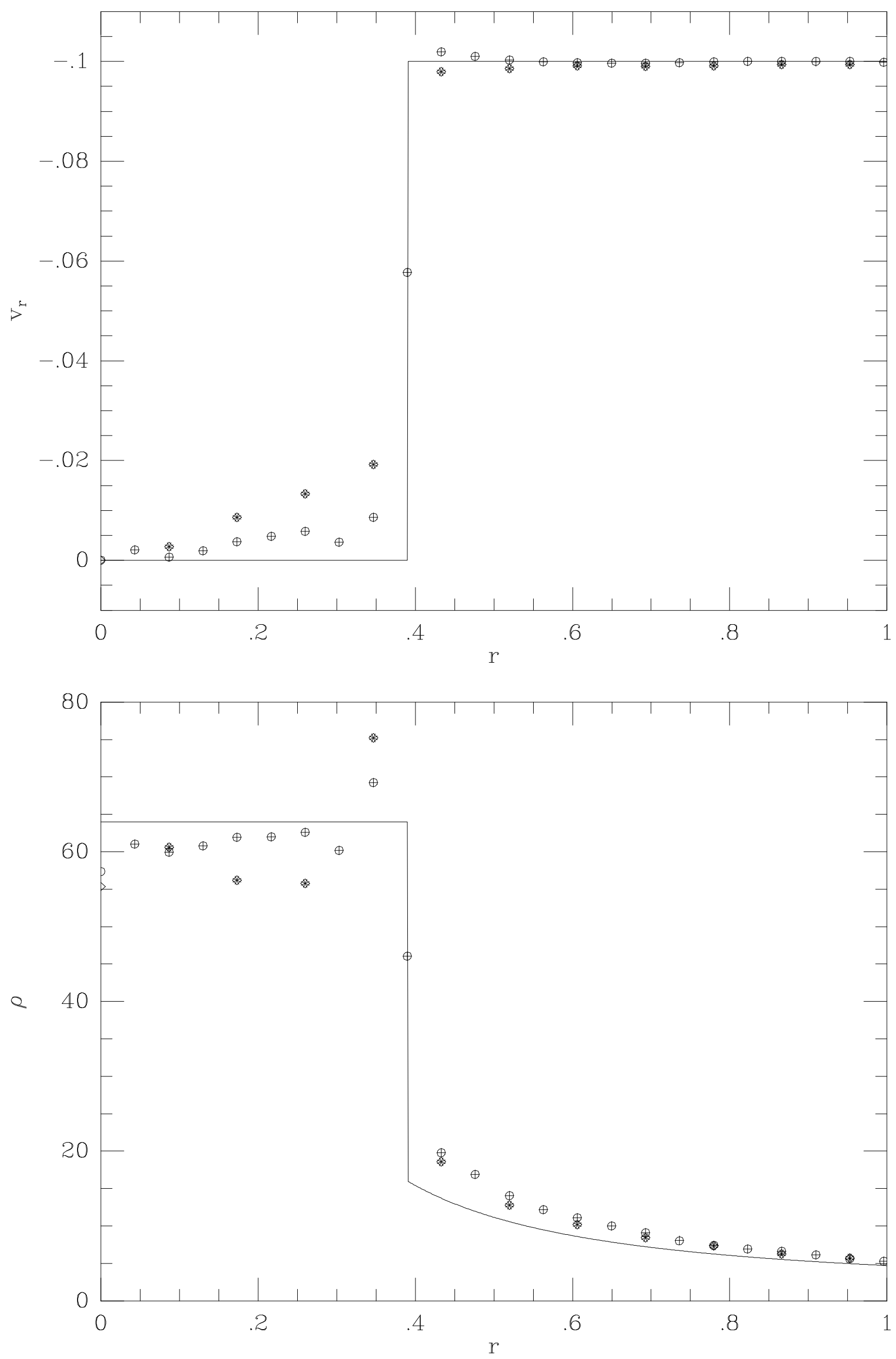


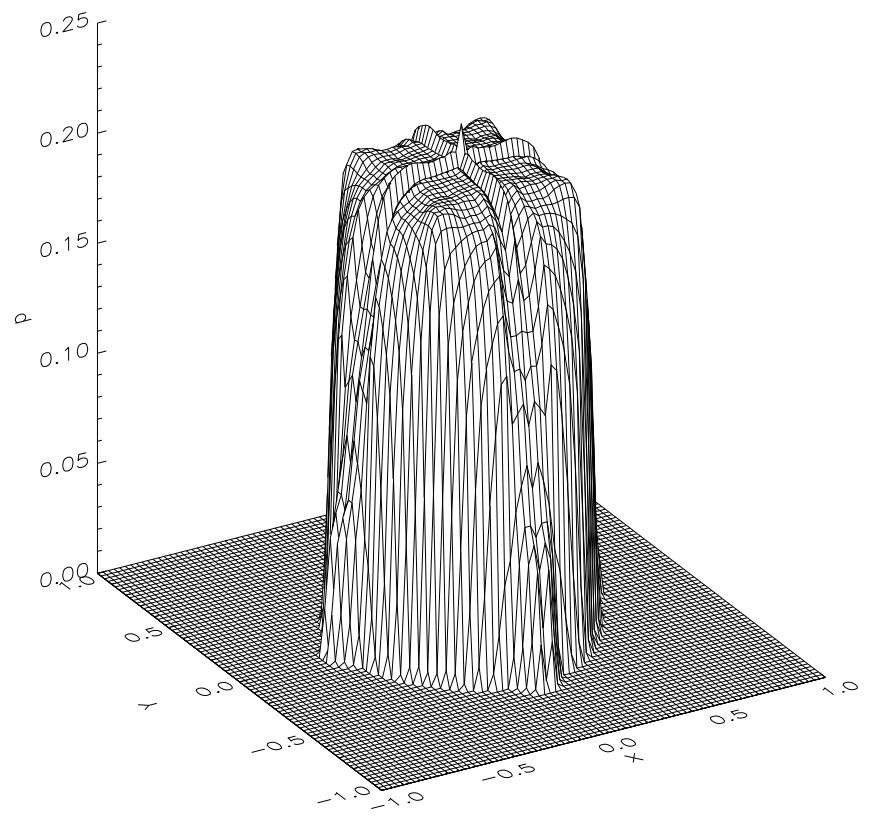



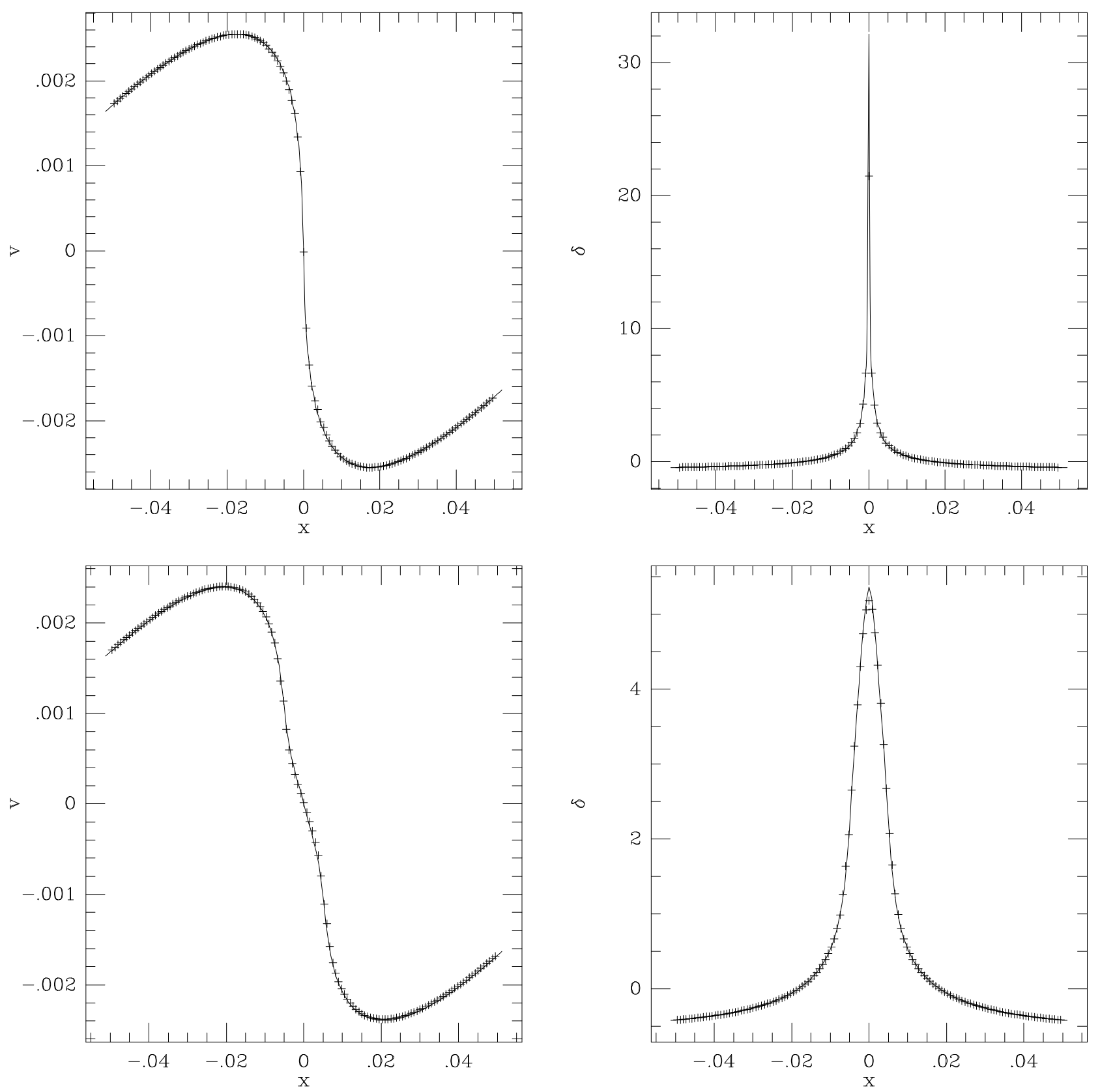

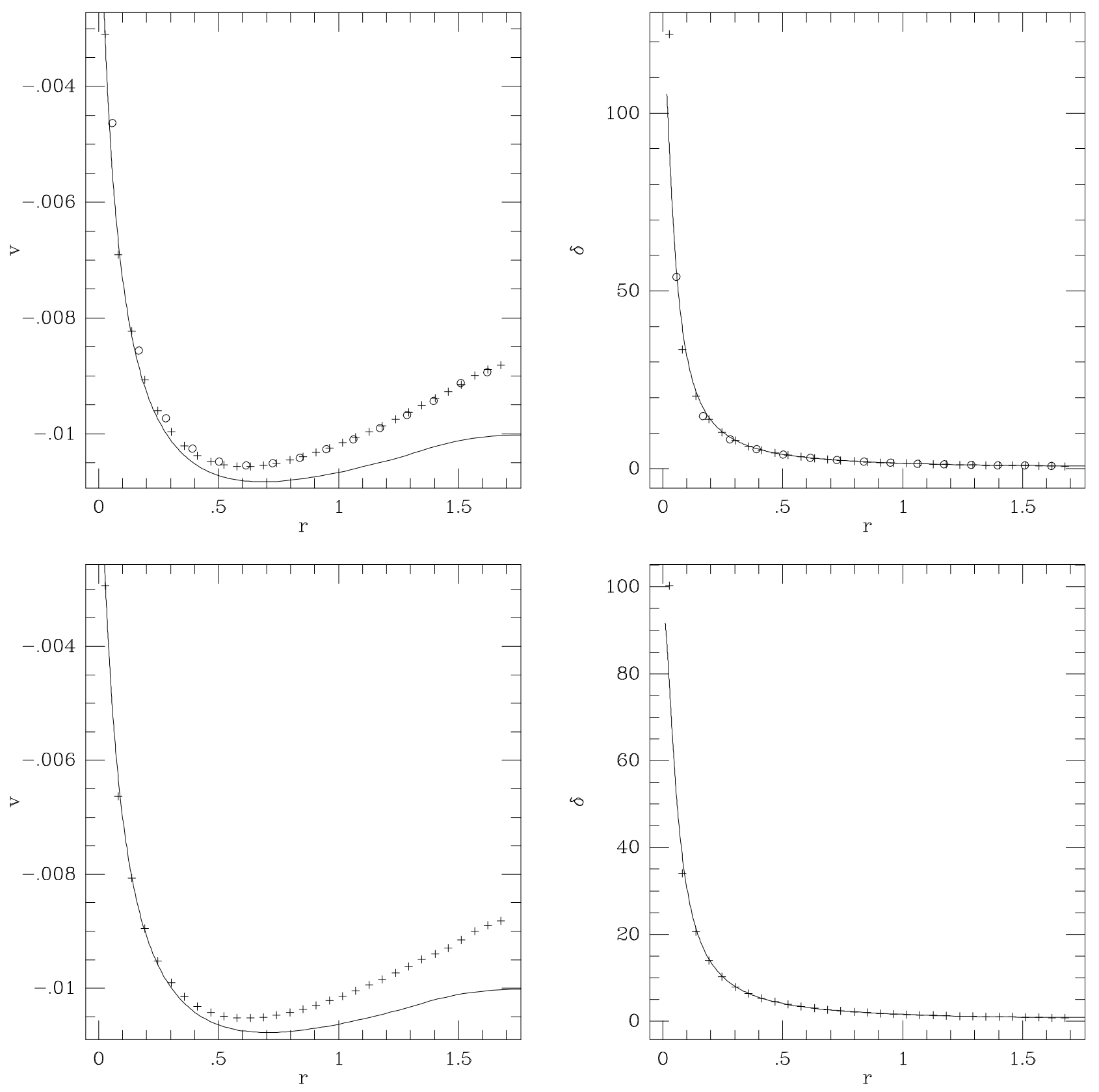